\title{
Prognostic Implications of Autophagy-Related Gene Signatures in Ewing's Sarcoma
}

\section{Yangfan Zhou}

The First Affiliated Hospital of Anhui Medical University

\section{Shusheng Wu}

The First Affiliated Hospital of USTC: Anhui Provincial Hospital

\section{Yulian Liu}

First Affiliated Hospital of Anhui Medical University

Bin Xu ( $\square$ youchen100@126.com)

First Affiliated Hospital of Anhui Medical University https://orcid.org/0000-0003-3103-4169

\section{Research}

Keywords: ewing'sarcoma, autophagy, gene signature, prognosis

Posted Date: March 22nd, 2021

DOl: https://doi.org/10.21203/rs.3.rs-342122/v1

License: (c) (i) This work is licensed under a Creative Commons Attribution 4.0 International License. Read Full License 


\section{Abstract}

Backgrand囚Ewing's sarcoma (ES) is a highly aggressive malignant bone tumor with a high incidence among children and adolescents. While autophagy often plays an important role in tumor development species, we developed an autophagy-related prognostic signature based on the GEO dataset.

Methods: Using the GEO database with the online website Human Autophagy Database(HADb), we screened for autophagy-related differential genes and we performed enrichment analysis and PPI analysis for autophagy-related differential genes, and then, constructed our autophagy-related prognostic gene signature by univariate Cox regression, lasso regression, and multivariate Cox regression .

Results: We screened a total of 72 autophagy-related differential genes, and a total of 8 autophagyrelated prognostic genes were screened by machine learning algorithms. ROC curves and survival curves also showed good predictive power of the 8 gene signatures. In addition, univariate and multivariate cox regression also showed that the 8 gene signatures were independent prognostic factors. And the results of GSEA enrichment analysis also revealed the correlation between autophagy and metabolic pathways.

Conlusion冈In summary, we developed an autophagy-related mRNA signature consisting of 8 mRNAs that effectively classified ES patients into low- and high-risk groups. The application of the signature in clinical treatment needs further observation to validate the validity of our findings.

\section{Introduction}

Ewing's sarcoma (ES) is a highly aggressive malignant bone tumor with a high incidence among children and adolescents[1, 2]. Moreover, ES has a high metastasis rate. According to statistical analysis, at the time of diagnosis, $20 \%-25 \%$ of patients already have lung metastases $(70-80 \%)$ and or bone metastases (40-50\%)[3] . Although the recurrence rate of ES is not high, the prognosis of relapsed ES patients is very terrible. In different studies, the 5 -year overall survival rate (OS) ranges from $8 \%$ to $15 \%[4]$. The molecular mechanism of the development stage and prognosis of ES is not completely clear, therefor it is necessary to explore the prognostic factors and therapeutic targets of ES.

Autophagy is the process of transporting damaged, denatured, or aged proteins and organelles to lysosomes for digestion and degradation[5]. Autophagy also plays such a double-sided role in tumors. Under normal physiological conditions, autophagy is beneficial to maintaining the self-stable state of cells. Autophagy can also prevent the accumulation of cancer-causing damaged proteins and organelles, and inhibit the carcinogenesis of cells[6, 7]. Autophagy also plays such a double-sided role in tumors. Under normal physiological conditions, autophagy is beneficial to maintaining the self-stable state of cells. Autophagy can also prevent the accumulation of cancer-causing damaged proteins and organelles, and inhibit the carcinogenesis of cells[5,6]. Thus, in most cases, autophagy is thought to inhibit the development of early tumors and promote the development of already formed tumors[8]. This suggests that regulating autophagy may be an effective intervention for cancer treatment[9]. In the past studies of ES, it has been reported that autophagy-related genes are closely related to the development of ES. For 
example, TRIM3 negatively regulates autophagy by promoting the degradation of Beclin 1 in ES cells, eWS-FLI1 actively regulates autophagy by increasing the expression of ATG4B in ES cells [11]. However, the relationship between autophagy-related genes and the prognosis of ES patients is still unknown.

Therefore, in our study, we first explored autophagy-related differential genes through the GEO database, then screened autophagy-related prognostic gene signatures by univariate cox regression, least absolute shrinkage and selection operator (LASSO) regression and multivariate cox regression, classified high- and low-risk groups, and validated our autophagy-related prognostic gene signatures by survival curves with receiver operating characteristic curve (ROC) curves. Finally, we further explored the underlying mechanisms by enrichment analysis of the high- and low-risk groups.

\section{Methods}

\section{1.collection of datasets}

In this study, we downloaded the ES dataset from the NCBI gene expression Omnibus (Geo) (http://www.ncbi.nlm.nih.gov/geo) database. The accession numbers are GSE17674 and GSE63157. Among them, GSE63157 is composed of two subgroups, which are GSE63155 and GSE63156. The data platform numbers of GSE17674 is GPL570 ( [HG-U133_Plus_2] Affymetrix Human Genome U133 Plus 2.0 Array ), and the data platform numbers of GSE63157 is GPL5175 ([HuEx-1_0-st] Affymetrix Human Exon 1.0 ST Array [transcript (gene) version] ). Among them, GSE17674 contains RNA-sequencing data and clinical survival information of $44 \mathrm{ES}$ samples, and RNA-sequencing data of 18 normal human skeletal muscle samples. GSE63157 contains RNA-sequencing data and clinical data of 85 ES samples. "rstudio" software and "Bioconductor" packages (http://www.bioconductor.org/) were used in the data analyses. Then, we remove the batch effect through R package named "sva" to normalize the datasets that we choosed(Fig1 A,B).In addition, we used 57 ES samples from the International Cancer Genome Consortium (ICGC; https://icgc.org) ICGC for external verification of prognostic genes.

\section{Screening for autophagy-related genes}

We downloaded the autophagy-related gene set through the website Human Autophagy Database (HADb) (http://www.autophagy.lu/) with 232 autophagy-related genes, next we used the "limma" R package and screened the differentially expressed genes in 18 cases of normal skeletal muscle and 129 cases of ES, setting The criteria were ( $\log F C>0.5, F D R<0.05)$, and finally, we selected the intersection of differential genes and autophagy-related genes by Venn analysis.

3. enrichment analysis and PPI network

We used the "org.Hs.eg.db" R package to enrichment analysis for autophagy-related differential genes and the "ggplot2" R package was used to plot the circles of GO analysis and the histograms of KEGG analysis. In addition, we obtained PPI reciprocal network graphs of autophagy-related differential genes 
from the "String" online website (https://string-db.org/) and optimized the graphs using "Cytoscape" software.

\section{Screening for prognosis-related autophagy genes}

First, a preliminary screening of autophagy-related differential genes was performed by univariate Cox regression with a screening criterion of $(P<0.05)$. After that, the screened genes were subjected to lasso regression, which was first proposed by Robert Tibshirani in 1996 and is known as Least absolute shrinkage and selection operator. The method is a compression estimation. It obtains a more refined model by constructing a penalty function that makes it compress some regression coefficients, i.e., force the sum of the absolute values of the coefficients to be less than some fixed value; while setting some regression coefficients to zero. Thus, it retains the advantage of subset shrinkage and is a biased estimator dealing with data with complex covariance. Finally, we performed multivariate Cox regression on the data screened by lasso regression and optimized the model again using the "step" function, and constructed the prognostic marker formula for ES based on the expression of autophagy-related genes and their correlation coefficients from the multivariate cox regression analysis as follows: risk The risk score $=\sum \sum$ icoefficient (genei) $\times$ expression(genei) was constructed, and then the median of the risk score was used to divide the samples into high-risk and low-risk groups.

Univariate and multivariate cox regression analyses were performed with a limited number of clinical variables based on risk score and used to confirm whether our prognostic genes were independent prognostic factors.

\section{5, GSEA enrichment analysis}

The R package "clusterprofiler" was used to perform GSEA enrichment analysis for the high and low-risk groups, and "enrichplot" was used to generate the gene set enrichment map with annotations, where pvalue cutoff $=0.05$ was set as the screening criterion. The enrichment score of 90 metabolic pathways was calculated with single-sample gene set enrichment analysis (sSGSEA) in the "GSVA" R package. The set of metabolism-related genes was obtained from the online website KEGG pathway (https://www.genome.jp/kegg/).

\section{Results}

1.Screening of autophagy-related differential genes and enrichment analysis

In the GEO cohort, we screened 129 ES and 18 normal skeletal muscle samples for differential genes and obtained a total of 5760 differential genes. 72 autophagy-related differential genes were obtained by taking intersections with 232 autophagy-related genes obtained from the HADb Human Autophagy Database (Fig2), and plotting the volcano map of autophagy-related differential genes (Fig3). We mapped the PPI protein interaction network of 72 autophagy-related genes (Fig4). GO and KEGG pathway enrichment analysis was performed, and the results of $\mathrm{GO}$ enrichment analysis showed that autophagy- 
related differential genes were mostly enriched in macroautophagy, regulation of autophagy, cellular, response to external stimulus, autophagosome assembly, autophagosome organization, and so on(Fig5). KEGG pathway enrichment analysis indicated that the genes correlated with Autophagy - animal, Autophagy - other, Kaposi sarcoma-associated, herpesvirus infection, Apoptosis, Mitophagy - animal, and so on(Fig6).

2. Distinction and Evaluation of 8 autophagy-related genes Prognostic Signature for ES

We selected 129 samples containing clinical prognostic information for the next analysis of 72 autophagy-related differential genes. By univariate cox regression in over sruviva I(OS), we found that a total of 22 genes were strongly associated with prognosis $(P<0.05)$, of which 12 genes were prognostic protective factors and 10 genes were prognostic risk factors(Fig7). Then, 12 genes were screened by lasso regression analysis (Fig8 A, B). Finally, based on multivariate cox regression and optimized the model using the "step" function, 8 genes, including HDAC1, EIF4EBP1, DLC1, BECN1, ATG10, SIRT1, PINK1, CFLAR were included in the final model. The risk scoring formula is Riskscore=PINK1(expression)* $(-0.930699593)+H D A C 1$ (expression)*0.462381473+BECN1*(-0.897598075)+CFLAR(expression)* $(-0.679324292)+\mathrm{DLC} 1$ (expression)*0.4698522+EIF4EBP $1 * 0.50843151+A T G 10 *(-0.734256293)+\mathrm{SIRT} 1$ * $(-0.659263165)$. We risk scored these 8 genes based on their risk coefficients as well as expression and divided the samples into high-risk and low-risk groups according to the median cut-off value. KaplanMeier (K-M) curves showed that the high-risk group had a worse prognosis ( $p<8.185 \mathrm{e}-07)$ (Fig9 A). Risk curves and scatter plots show the risk scores and survival status of all patients with ES. The mortality rate and hazard ratio were lower in the low-risk group than in the high-risk group. Mortality and risk factors were lower in the low-risk group than in the high-risk group, and the Heatmap showed that HDAC1, EIF4EBP1, DLC1 were upregulated in the high-risk group, while other genes were significantly downregulated (Fig10 A, B, C). Besides, the time-dependent receiver operating characteristic curve (ROC) curve is used to evaluate the 8 autophagy-related gene signals in predicting the total 3 to 5 years of ES patients. Precision in terms of lifetime. The area under the ROC value (AUC) for 3 years and 5 years were 0.897 and 0.849 respectively (Fig9 B). We validated these 8 prognostic gene signatures in the ICGC cohort using the same formula and plotted risk curves, scatter plots and heatmap.(Fig11 A,B,C) The survival curves also reflected a significantly worse prognosis in the high-risk group $(p=0.05)$, with AUC values of 0.595 and 0.666 at 3 and 5 years, respectively, both reflecting the good predictive power of our model (Fig9 C,D).

3. Evaluation of 8 autophagy-associated prognostic genes as independent prognostic factors in GEO chohort

Combining clinical information age and sex, all eight autophagy-related prognostic genes signature could be used as independent prognostic factors in both univariate and multivariate cox regression $(P<0.01)$ (Fig12 A, B).

4. GSEA enrichment analysis 
To elucidate the biological pathways and functions associated with the risk score. We performed enrichment analysis on the high-risk group and the low-risk group. Interestingly, in the results of the enrichment analysis, there were enrichment results in the low-risk group concerning multiple metabolic pathways (Fig13), like heparin metabolic process $\triangle$ Histidine metabolism and Propanoate metabolism. To investigate the differences in metabolic pathways between high and low-risk groups, we used the metabolic pathway-related gene set obtained from the KEGG pathway online website, quantified the enrichment scores of 90 metabolic pathways by the R package "GSVA", and created box plots to visualize the differences in metabolic pathways between high and low-risk groups (Fig14), We found differences in a large number of metabolic pathways in both high and low-risk groups, including Mucin type 0-glycan biosynthesis, Other types of O-glycan biosynthesis, Glutathione metabolism, etc. were significantly enriched in the high-risk group, and then Starch and sucrose metabolism $₫$ beta-Alanine metabolism $₫$ Tryptophan metabolism, etc. were significantly enriched in the low-risk group. Among them, Histidine metabolism and Propanoate metabolism were also significantly different from the high-risk group $(P<0.001)$, which was consistent with the results of our GSEA enrichment analysis.

\section{Discussion}

The influence of autophagy on its development has been less addressed in past studies of ES, and although some autophagy-related genes have been studied, the prognostic association of autophagyrelated genes with ES patients is still unknown. In our study, we analyzed the expression of 232 autophagy-related genes in ES patients and finally identified 8 prognostic genes associated with autophagy, which may provide new ideas and directions for the treatment and research of ES.

Autophagy-related prognostic genes based on autophagy have been reported in colon, colorectal, plasma ovarian and non-small cell lung cancers [12-15], which illustrates the close association between autophagy and cancer development. The model we constructed included a total of 8 genes (HDAC1, EIF4EBP1, DLC1, BECN1, ATG10, SIRT1, PINK1, CFLAR). In recent years, the physiological role of HDAC1 has been explored in a variety of tumors, and silencing of HDAC1 in ovarian cancer improves chemotherapy response [16]. In glioma cells, inhibition of HDAC1 expression inhibits glioma cell invasion and induces apoptosis [17]. And in the past, HDAC1 was a commonly mutated gene in sarcoma along with TP53, NF1, and other genes [18], but its pathogenesis in ES needs to be investigated more deeply. EIF4EBP1 is a translation repressor protein encoded by a gene that competitively binds to eukaryotic translation initiation factor 4E (EIF4E), thereby inhibiting the assembly of the eIF4E complex and thus cap-dependent translation, thus initiating mRNA translation when EIF4EBP1 is downregulated, thereby promoting cell growth and proliferation [19]. A study found that DLC1 inhibits Wnt/ $\beta$-catenin signaling by reducing T-cell factor 4 (TCF4) expression and the interaction between $\beta$-catenin and TCF4, and suppresses autophagy by inhibiting the dissociation of ROCK1 and Beclin1-Bcl2 complexes in hepatocellular carcinoma cell lines [20]. However, the relevance of DLC1 to autophagy in ES is unclear. becN1 / Beclin1 is a central protein that assembles cofactors to form the BECN1-PIK3C3-PIK3R4 complex, which triggers the autophagic protein cascade response [21]. The ATG family tends to regulate the production of autophagic vesicles [22], while ATG10, a member of the ATG family, is also closely 
associated with tumorigenesis and development, e.g., high expression of ATG10 in colorectal cancer is associated with lymphovascular infiltration and lymph node metastasis, leading to tumor migration and invasion [23]. In addition, overexpression of ATG10 in cancers such as nasopharyngeal, hepatocellular, and gastric cancers has been associated with poor patient prognosis [24-26]. Past studies have found that overexpression of SIRT1 is associated with ES metastasis and poor prognosis, while the SIRT1 / 2 inhibitor Tenovin-6 kills ES cells in vitro [27]. The PINK1 gene is located on the short arm of chromosome 1 and encodes a 581 amino acid serine/threonine protein kinase [28]. Current studies have shown that PINK1 plays an important role in tumorigenesis and development by inducing autophagy to eliminate dysfunctional mitochondria [29]. In contrast, CFLAR has been relatively little studied in tumors, but its downregulation is often associated with enhanced autophagy of T cells and leads to enhanced cytoprotective effects of T cells [30], which may provide a new direction for ES research.

In addition, we found multiple metabolism-related pathways appeared in the results of our GSEA enrichment analysis. By ssGSEA analysis between high and low-risk groups, it may be possible to explain some of the relationships between autophagy and metabolism, in past studies, the following hypotheses were made for the association between autophagy, tumor, and metabolism, one hypothesis is that the maintenance of metabolic function through the supply of autophagic substrates is particularly critical for tumor growth [5]. Autophagy is also essential for the removal of damaged proteins and organelles, especially mitochondria. The second hypothesis is that tumor cells require autophagy not only to provide substrates for mitochondria, but also to protect the functional pool of mitochondria by removing defective mitochondria, which is essential to maintain metabolism and survival [5]. However, there still exist metabolic pathways that are protective factors for tumors, for example, the metabolic degradation of histidine increases the sensitivity of cancer cells to methotrexate, and this approach also helps to reduce the side effects of methotrexate treatment in mouse models [31]. Therefore, different treatment regimens should be adapted for different subgroups of patients.

However, our study still has some shortcomings, on the one hand, our study is based solely on the results of machine learning and bioinformatics analysis, lacking external data validation and experimental verification, and on the other hand, it lacks some key clinical information because ES is relatively rare and our study is based on public databases. In addition, more in-depth studies are still needed on the developmental mechanisms of these 8 autophagy-related prognostic genes in ES.

\section{Conlusion}

In summary, we developed an autophagy-related mRNA signature consisting of 8 mRNAs that effectively classified ES patients into low- and high-risk groups. The application of the signature in clinical treatment needs further observation to validate the validity of our findings.

\section{Declarations}

\section{Funding}


This research did not receive any specific grant from funding agencies in the public, commercial, or notfor-profit sectors.

\section{Compliance with ethical standards}

Conflict of interest No financial interests are to be disclosed by the authors.

\section{Availability of data and materials}

The data used to support the findings of this study are included within the article.

\section{Consent for publication}

All authors agree to publish the paper.

\section{Acknowledgments}

We want to thank GEO,R software for free use.

\section{Ethics statement}

This study was based on the data released from an online publicly available GEO database, and data extracted from GEO were identified as nonhuman study. Thus, it was deemed exempt by the Ethics Committee of the first affiliated hospital of anhui medical university (Hefei, China).

\section{Contributions}

Yangfan Zhou master1 :Data processing, Article writing.

Shusheng Wu doctor1 :Provide data ,Data processing, artical writing.

Guanxiong Wang master2 : artical writing

Bin Xu doctor : provide data

\section{References}

1. Balamuth NJ. Womer RB. Ewing's sarcoma. Lancet Oncol. 2010;11(2):184-92.

2. Gaspar N. Hawkins DS, Dirksen U, et al Ewing Sarcoma: Current Management and Future Approaches Through Collaboration. J Clin Oncol. 2015;33(27):3036-46.

3. Bosma SE. Rueten-Budde AJ, Lancia C, et al Individual risk evaluation for local recurrence and distant metastasis in Ewing sarcoma: A multistate model: A multistate model for Ewing sarcoma. Pediatr Blood Cancer. 2019;66(11):e27943.

4. Palmerini E. Jones $\mathrm{RL}$, Setola $\mathrm{E}$, et al Irinotecan and temozolomide in recurrent Ewing sarcoma: an analysis in 51 adult and pediatric patients. Acta Oncol. 2018;57(7):958-64. 
5. Guo JY. Xia B, White E. Autophagy-mediated tumor promotion. Cell. 2013;155(6):1216-9.

6. Amaravadi RK. Kimmelman AC, Debnath J. Targeting Autophagy in Cancer: Recent Advances and Future Directions. Cancer Discov. 2019;9(9):1167-81.

7. Glick D. Barth S, Macleod KF. Autophagy: cellular and molecular mechanisms. J Pathol. 2010;221(1):3-12.

8. White E. Mehnert JM, Chan CS. Autophagy, Metabolism, and Cancer. Clin Cancer Res. 2015;21(22):5037-46.

9. Li X. He S, Ma B. Autophagy and autophagy-related proteins in cancer. Mol Cancer. 2020;19(1):12. Published 2020 Jan 22.

10. Lu Q. Zhang Y, Ma L, et al TRIM3 Negatively Regulates Autophagy Through Promoting Degradation of Beclin1 in Ewing Sarcoma Cells. Onco Targets Ther. 2019;12:11587-95. Published 2019 Dec 30.

11. Lu Q. Zhang $Y, M a ~ L$, et al EWS-FLI1 positively regulates autophagy by increasing ATG4B expression in Ewing sarcoma cells. Int J Mol Med. 2017;40(4):1217-25.

12. Mo S. Dai W, Xiang W, et al Prognostic and predictive value of an autophagy-related signature for early relapse in stages I-III colon cancer. Carcinogenesis. 2019;40(7):861-70.

13. Zhou Z. Mo S, Dai W, et al Development and Validation of an Autophagy Score Signature for the Prediction of Post-operative Survival in Colorectal Cancer. Front Oncol. 2019;9:878. Published 2019 Sep 9.

14. An Y. Bi F, You Y. Liu X, Yang Q. Development of a Novel Autophagy-related Prognostic Signature for Serous Ovarian Cancer. J Cancer. 2018;9(21):4058-71. Published 2018 Oct 18.

15. Liu Y. Wu L, Ao H, et al Prognostic implications of autophagy-associated gene signatures in nonsmall cell lung cancer. Aging. 2019;11(23):11440-62.

16. Liu X. Yu Y, Zhang J, et al HDAC1 Silencing in Ovarian Cancer Enhances the Chemotherapy Response. Cell Physiol Biochem. 2018;48(4):1505-18.

17. Wang XQ. Bai HM, Li ST, et al Knockdown of HDAC1 expression suppresses invasion and induces apoptosis in glioma cells. Oncotarget. 2017;8(29):48027-40.

18. Teicher BA. Searching for molecular targets in sarcoma. Biochem Pharmacol. 2012;84(1):1-10.

19. Cha YL. Li PD, Yuan LJ, et al EIF4EBP1 overexpression is associated with poor survival and disease progression in patients with hepatocellular carcinoma. PLoS One. 2015;10(2):e0117493. Published 2015 Feb 6.

20. Wu HT. Xie CR, Lv J, et al The tumor suppressor DLC1 inhibits cancer progression and oncogenic autophagy in hepatocellular carcinoma. Lab Invest. 2018;98(8):1014-24.

21. Han T. Guo M, Gan M. Yu B, Tian X. Wang JB. TRIM59 regulates autophagy through modulating both the transcription and the ubiquitination of BECN1. Autophagy. 2018;14(12):2035-48.

22. Mizushima N. Yoshimori T, Ohsumi Y. The role of Atg proteins in autophagosome formation. Annu Rev Cell Dev Biol. 2011;27:107-32. 
23. Jo YK. Kim SC, Park IJ, et al Increased expression of ATG10 in colorectal cancer is associated with lymphovascular invasion and lymph node metastasis. PLoS One. 2012;7(12):e52705.

24. Shen M. Lin L. Functional variants of autophagy-related genes are associated with the development of hepatocellular carcinoma. Life Sci. 2019;235:116675.

25. Yang Z. Liu Z. Potentially functional variants of autophagy-related genes are associated with the efficacy and toxicity of radiotherapy in patients with nasopharyngeal carcinoma. Mol Genet Genomic Med. 2019;7(12):e1030.

26. Wu M. Chen B, Pan X. Su J. Prognostic Value of Autophagy-related Proteins in Human Gastric Cancer. Cancer Manag Res. 2020;12:13527-40. Published 2020 Dec 31.

27. Ban J. Aryee DN, Fourtouna A, et al Suppression of deacetylase SIRT1 mediates tumor-suppressive NOTCH response and offers a novel treatment option in metastatic Ewing sarcoma. Cancer Res. 2014;74(22):6578-88.

28. Matsuda S. Kitagishi Y, Kobayashi M. Function and characteristics of PINK1 in mitochondria. Oxid Med Cell Longev. 2013;2013:601587.

29. Lu X. Liu QX, Zhang J, et al PINK1 Overexpression Promotes Cell Migration and Proliferation via Regulation of Autophagy and Predicts a Poor Prognosis in Lung Cancer Cases. Cancer Manag Res. 2020;12:7703-14. Published 2020 Aug 24.

30. He MX. He YW. CFLAR/c-FLIPL: a star in the autophagy, apoptosis and necroptosis alliance. Autophagy. 2013;9(5):791-3.

31. Frezza C. Histidine metabolism boosts cancer therapy. Nature. 2018;559(7715):484-5.

\section{Figures}

A

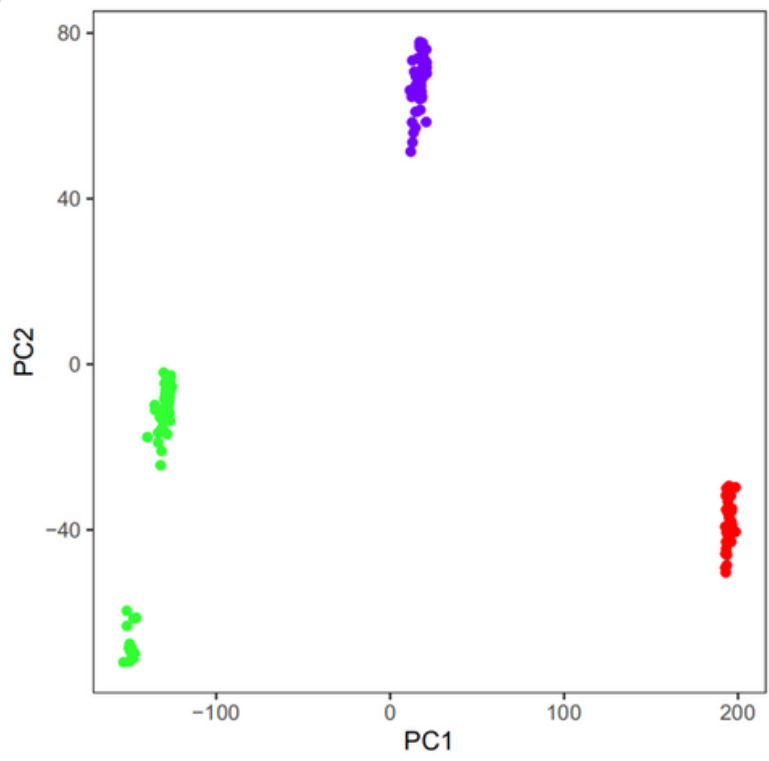

B

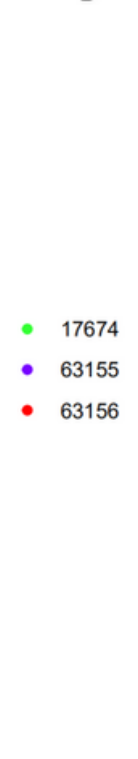

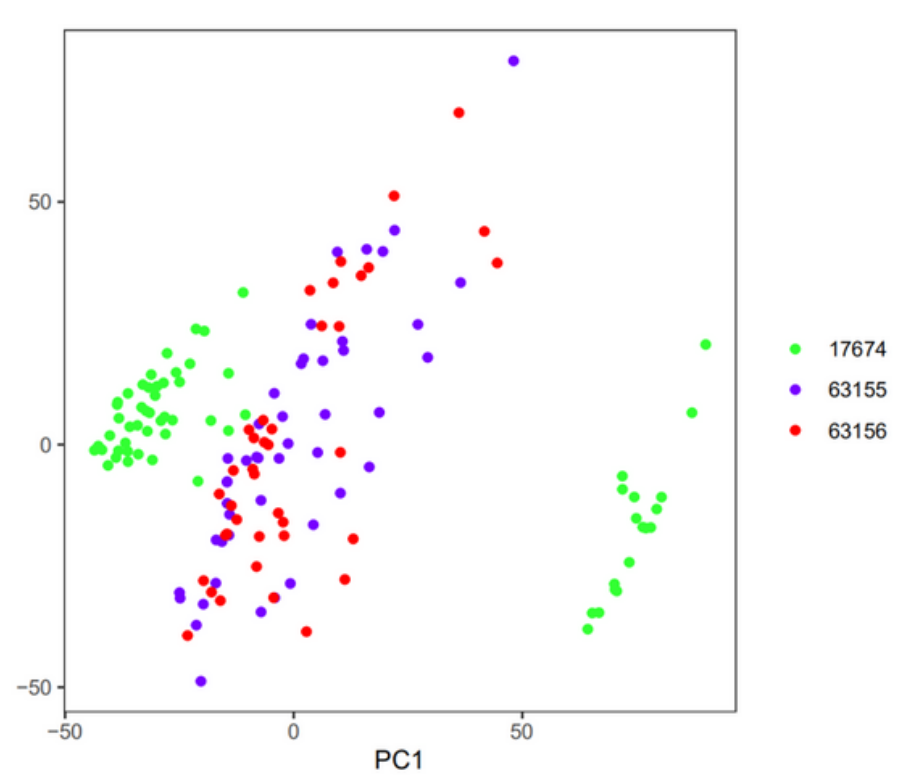

Figure 1 
The points of the scatter plots visualize the samples based on the top two principal components (PC1 and PC2) of gene expression profiles without (A) and with (B) the removal of batch effect.

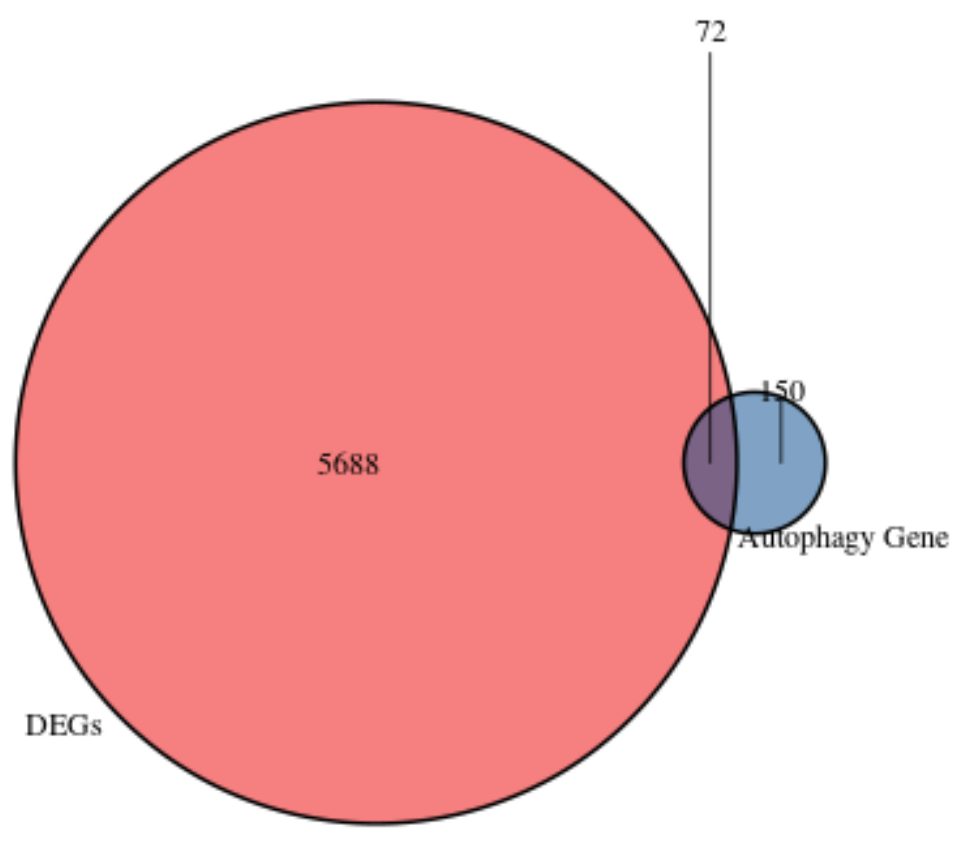

Figure 2

Venn analysis was used to study the Autophagy-related genes. 


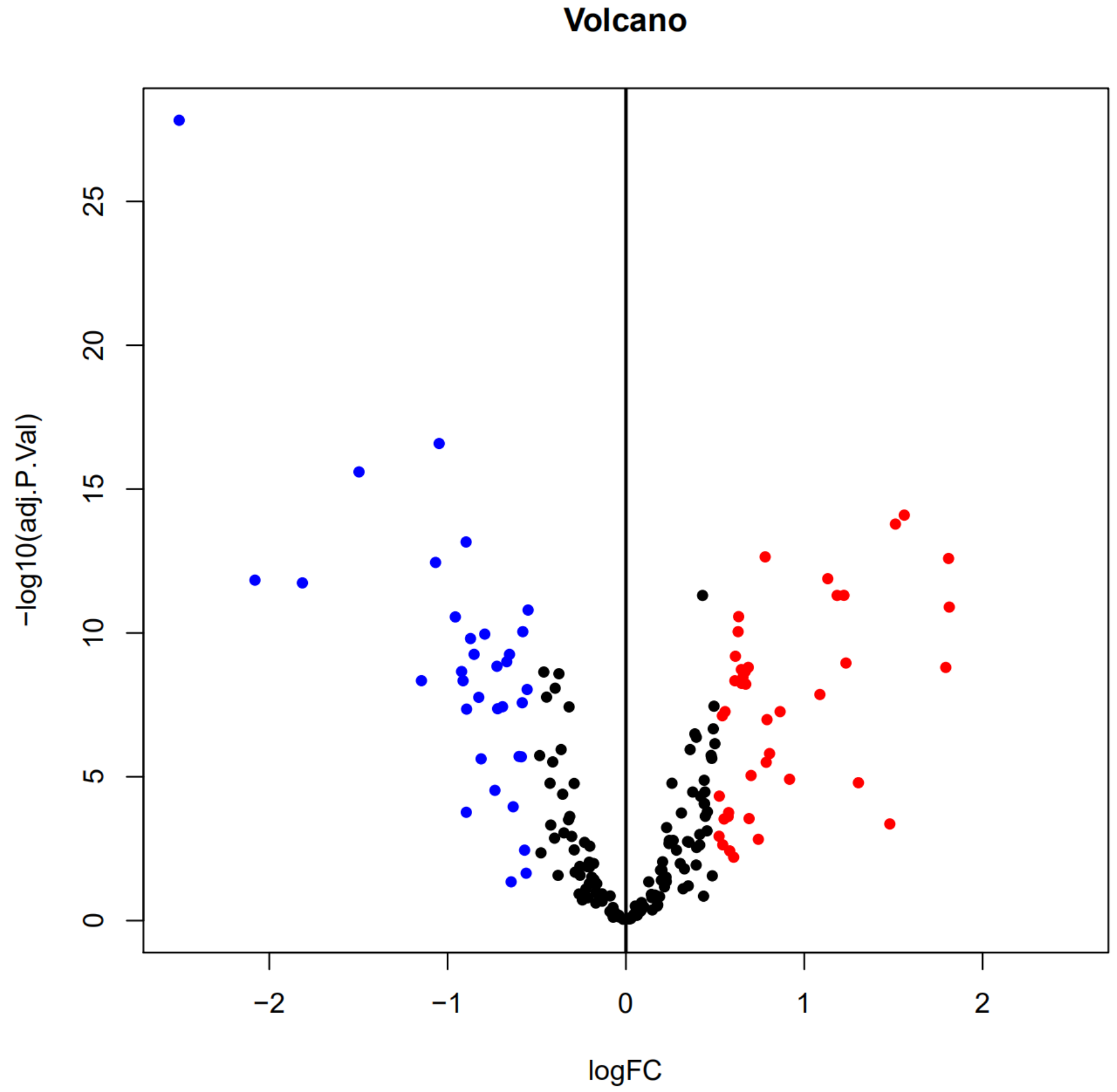

Figure 3

Volcano map demonstrates autophagy-related differential genes 


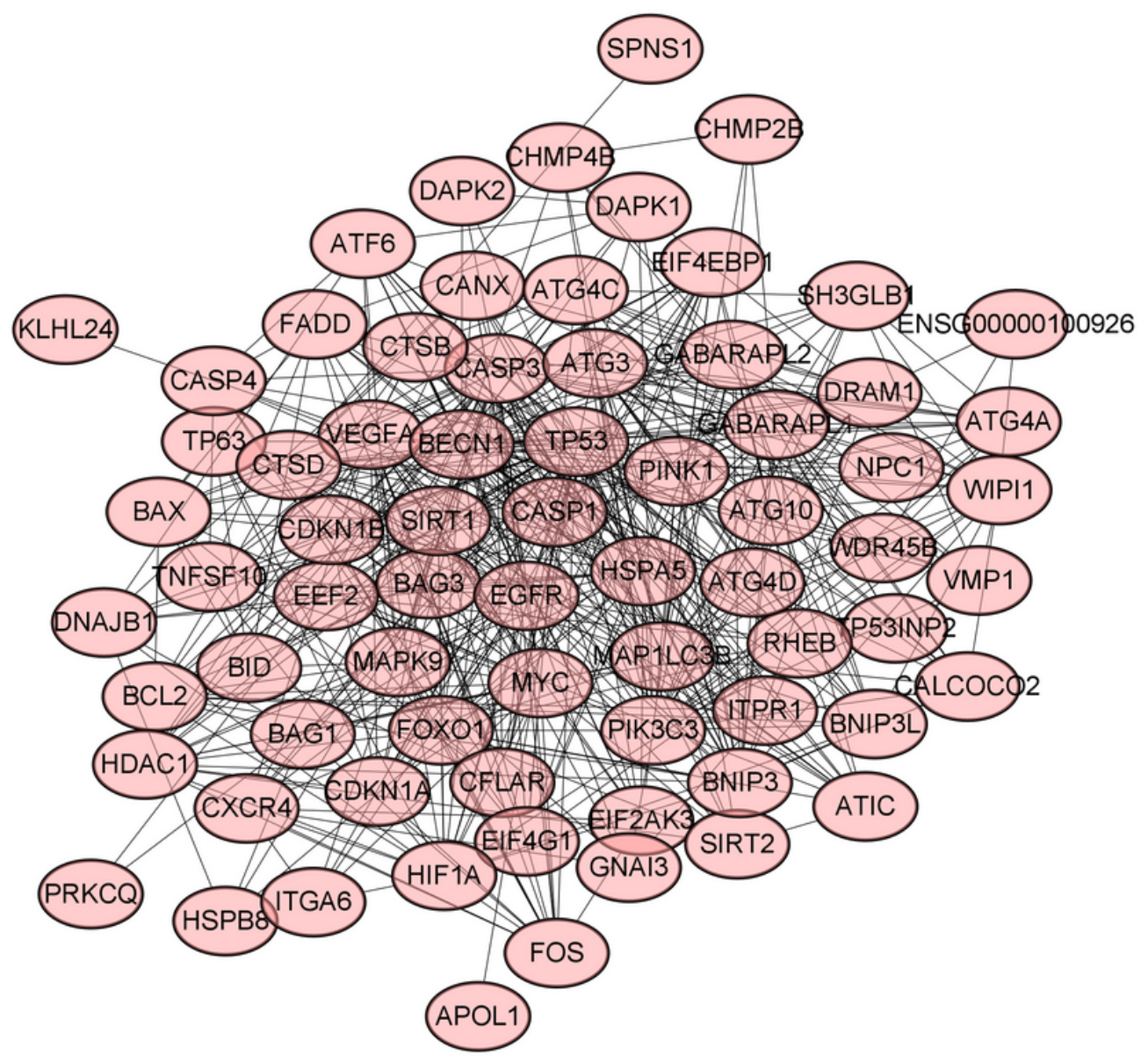

Figure 4

Interaction network map of PPI proteins with autophagy-related differential genes 

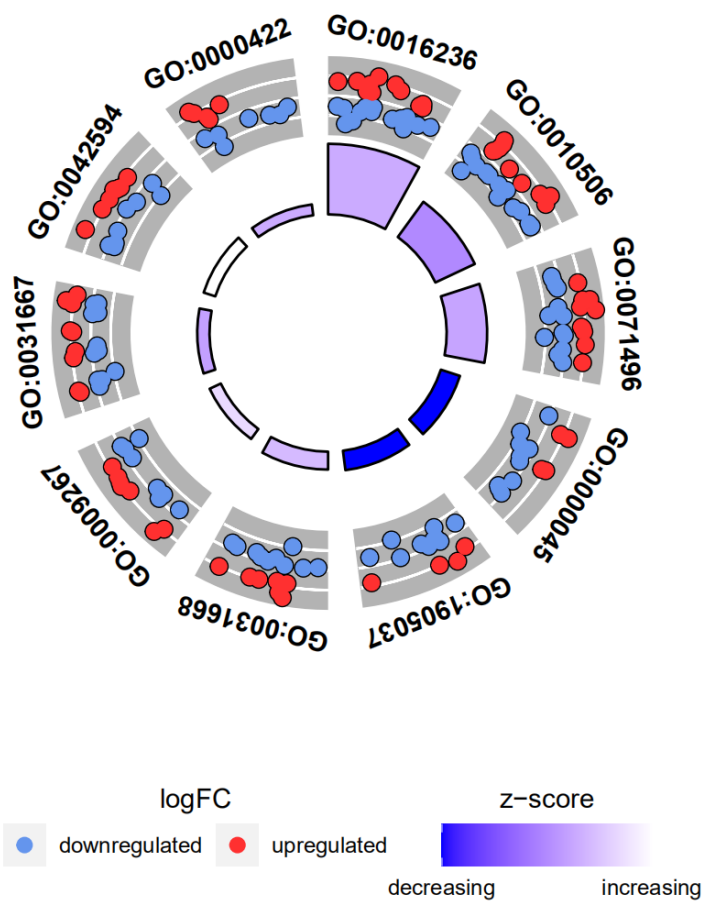

\begin{tabular}{|c|r|}
\hline ID & $\begin{array}{c}\text { Description } \\
\text { macroautophagy }\end{array}$ \\
\hline GO:0016236 & $\begin{array}{r}\text { regulation of autophagy } \\
\hline \text { GO:0010506 }\end{array}$ \\
\hline GO:0071496 & cellular response to external stimulus \\
\hline GO:0000045 & autophagosome assembly \\
\hline GO:1905037 & autophagosome organization \\
\hline GO:0031668 & cellular response to extracellular stimulus \\
\hline GO:0009267 & cellular response to starvation \\
\hline GO:0031667 & response to nutrient levels \\
\hline GO:0042594 & response to starvation \\
\hline GO:0000422 & autophagy of mitochondrion \\
\hline
\end{tabular}

\section{Figure 5}

Circle diagram of GO enrichment analysis 


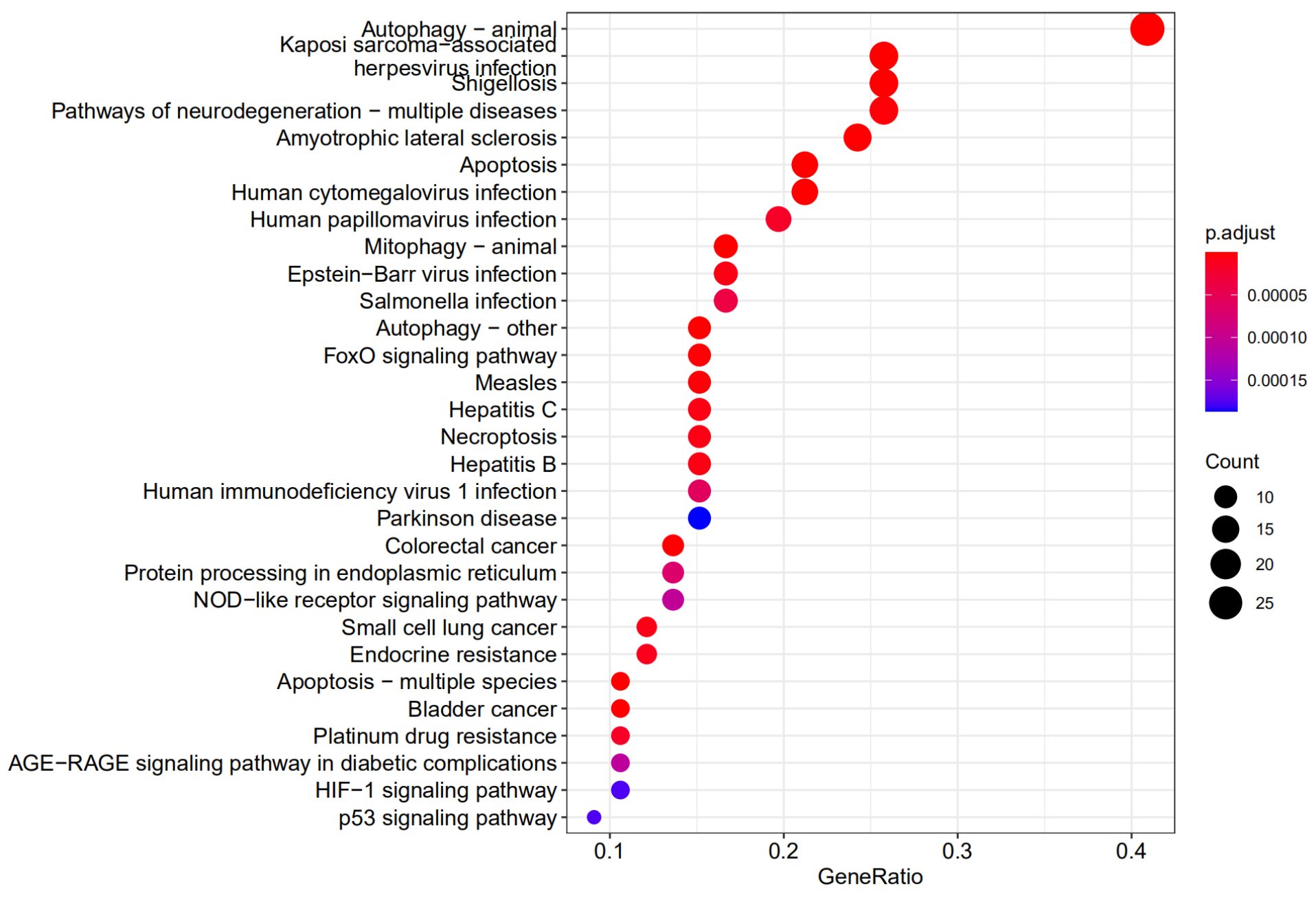

\section{Figure 6}

Dot plot of KEGG enrichment analysis 


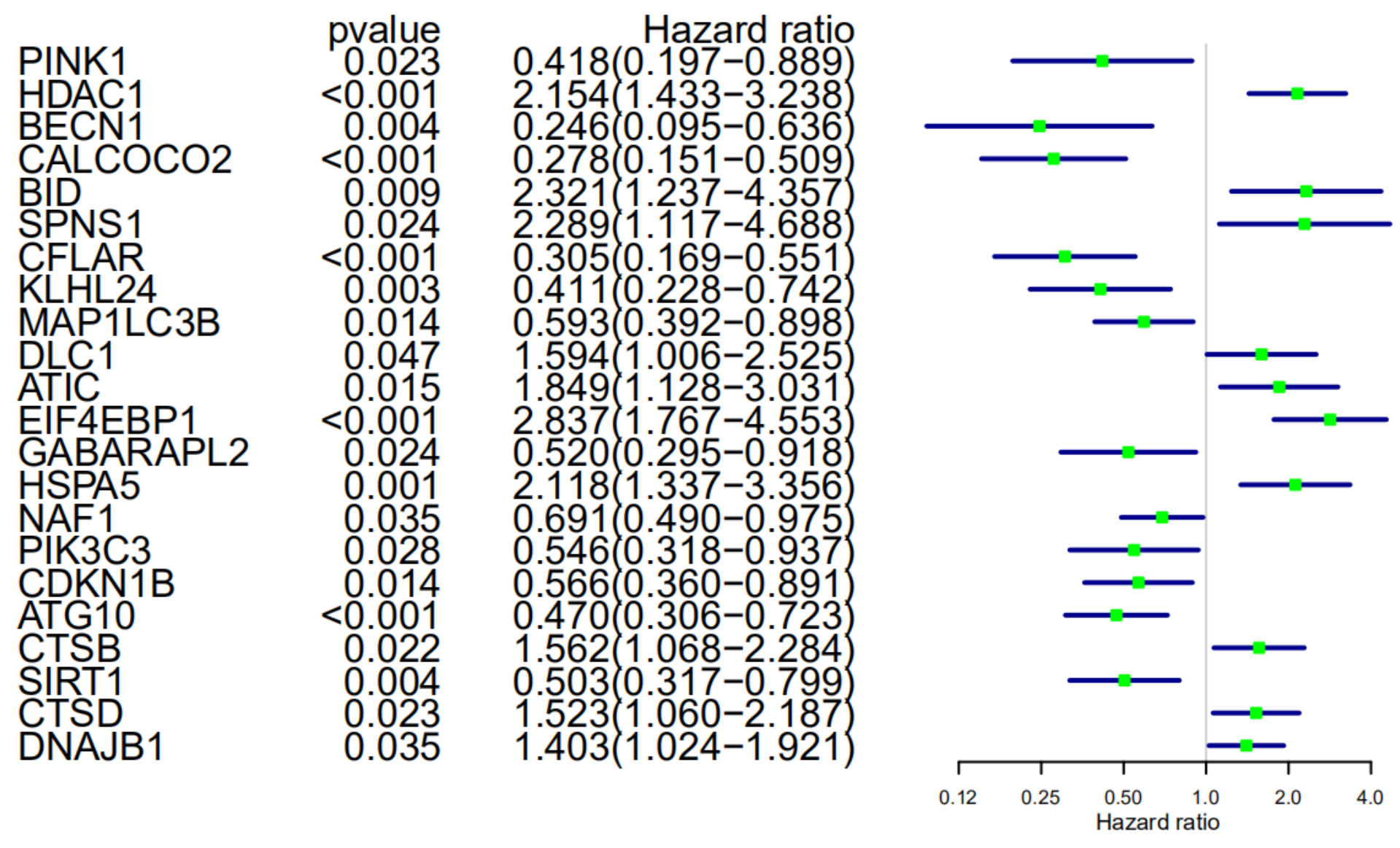

Figure 7

Genes screened by one-way cox regression $(p<0.05)$ A
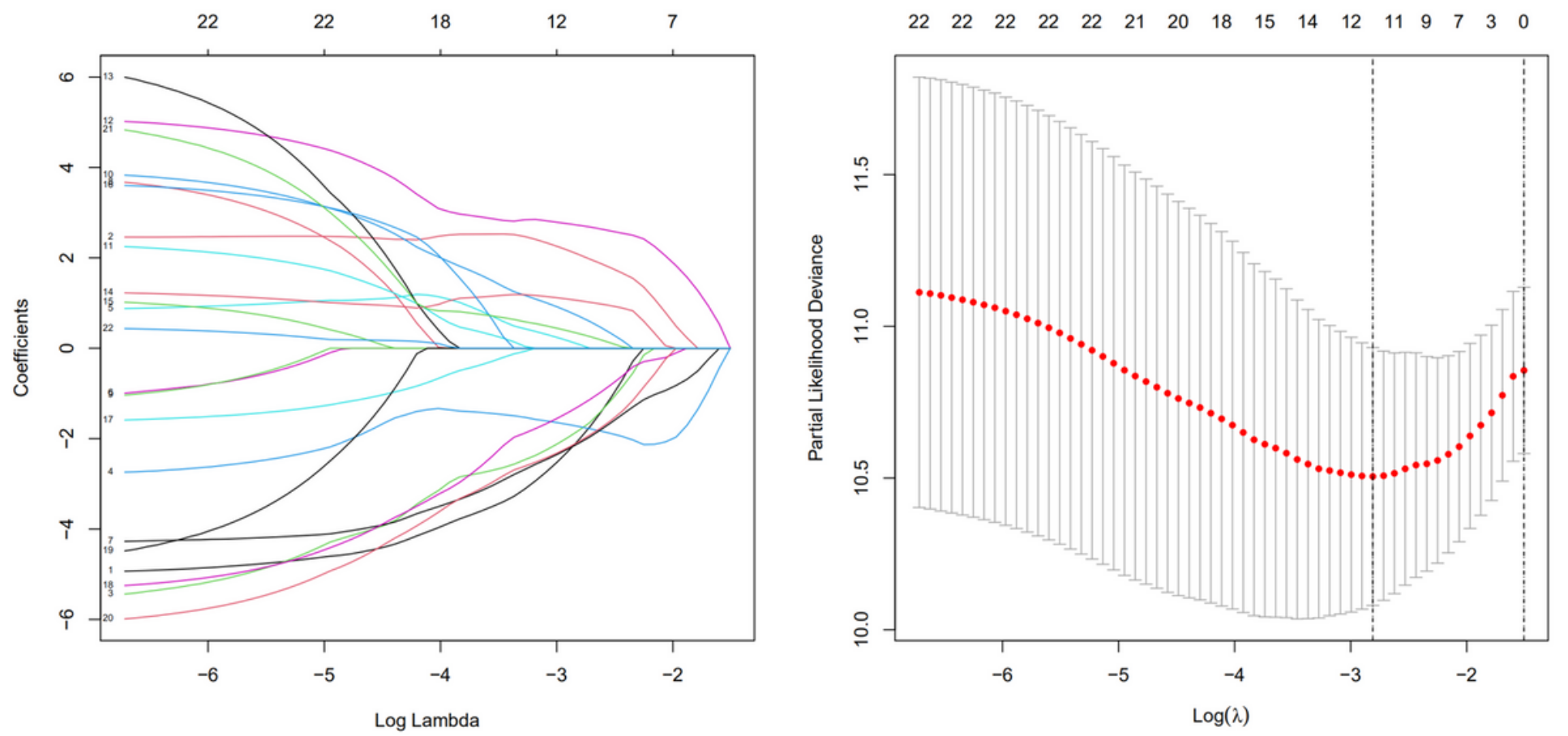


\section{Figure 8}

The LASSO Cox analysis identified 11 genes associated with prognosis and the optimal values of the penalty parameter were defined by 1,000-round cross-validation.

A

Risk $\leftarrow$ High risk $\leftarrow$ Low risk

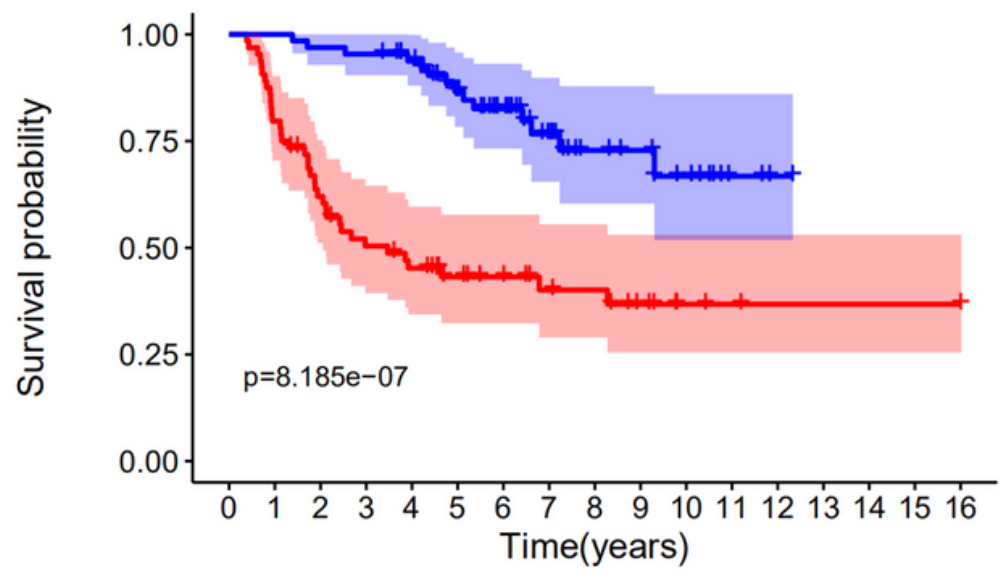

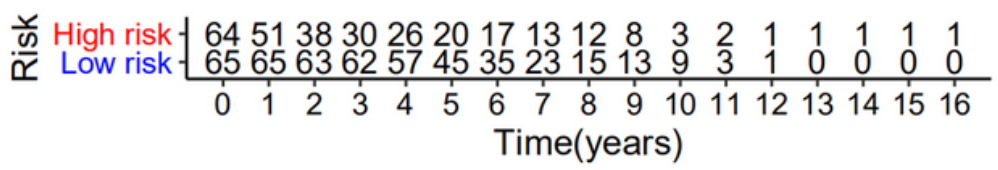

C

Risk + High risk + Low risk

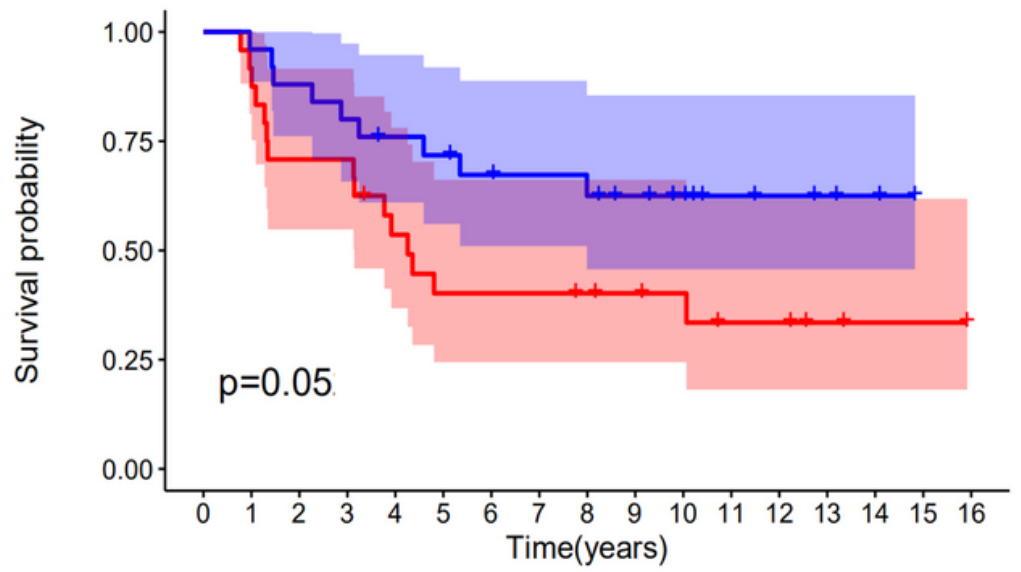

\begin{tabular}{l|lllllllllllllllll} 
w. High risk & 24 & 22 & 17 & 17 & 12 & 9 & 9 & 9 & 8 & 7 & 6 & 4 & 4 & 2 & 1 & 1 & 0
\end{tabular}

$\dot{\bar{\alpha}}$ Low risk \begin{tabular}{ccccccccccccccccc}
25 & 24 & 22 & 20 & 18 & 17 & 15 & 14 & 13 & 11 & 8 & 5 & 4 & 3 & 2 & 0 & 0 \\
\hline 0 & 1 & 2 & 3 & 4 & 5 & 6 & 7 & 8 & 9 & 10 & 11 & 12 & 13 & 14 & 15 & 16
\end{tabular} Time(years)

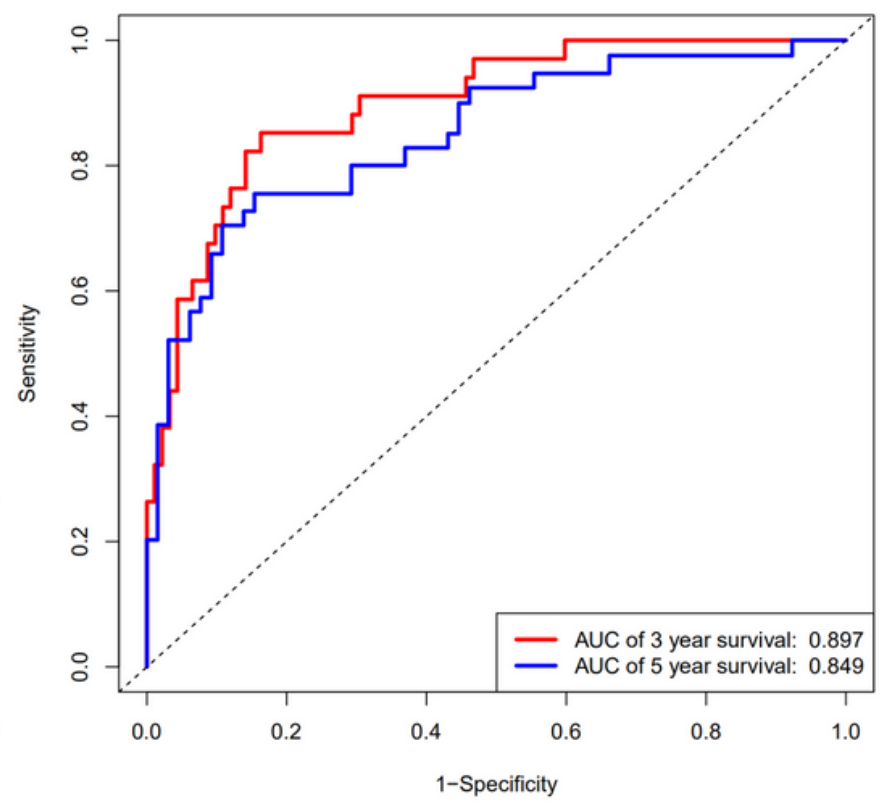

D

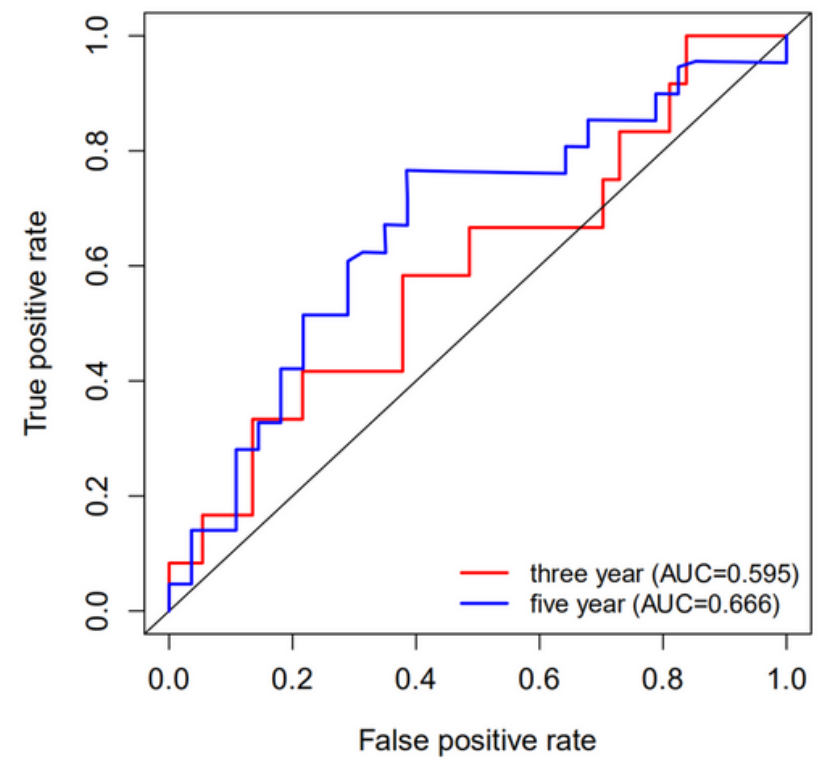

\section{Figure 9}

The survival curve $(A)$ show that low risk level has better prognosis in GEO cohort, the time-dependent $\mathrm{ROC}(\mathrm{B})$ curves of the prognostic gene signature in GEO cohort. The survival curve(C) show that low risk level has better prognosis in ICGC cohort, the time-dependent ROC(D) curves of the prognostic gene signature in ICGC cohort. 
A

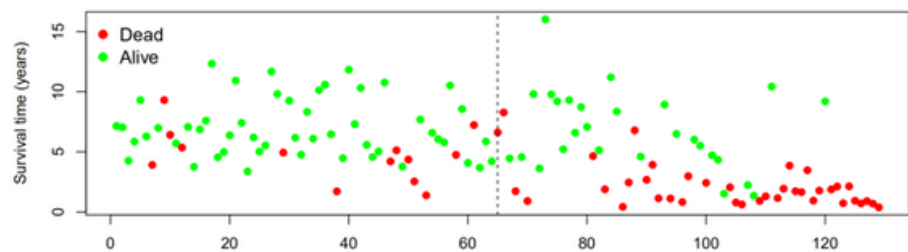

B

Patients (increasing risk socre)
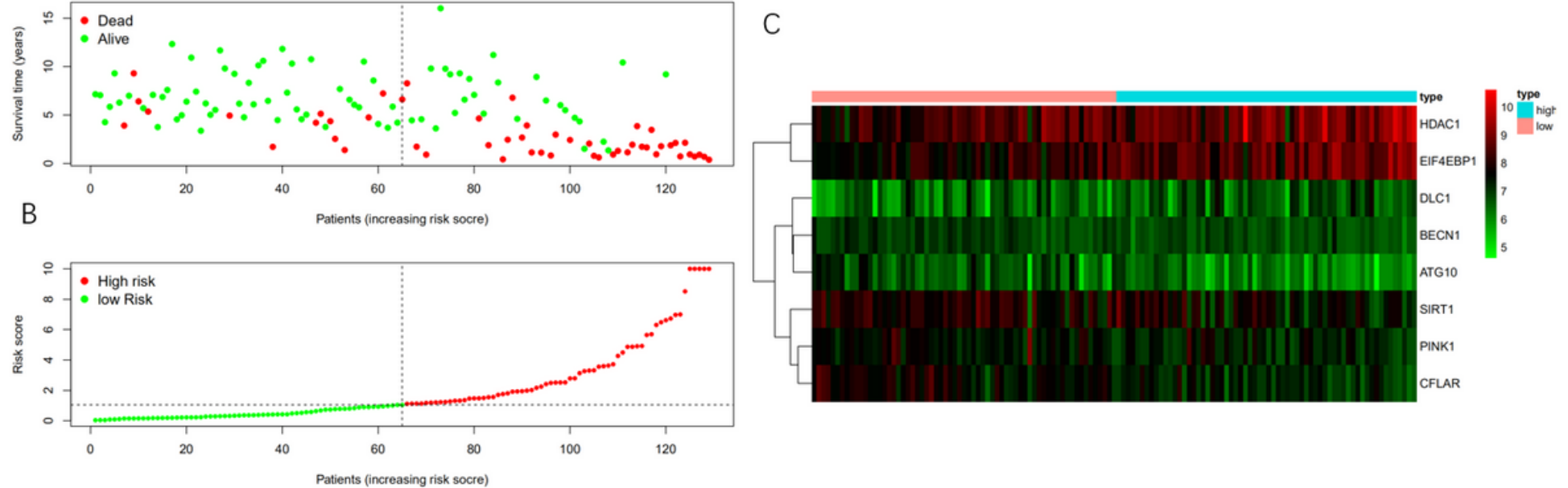

Figure 10

(A)The distribution s of the survival time, and status of patients, (B)The distributions of the risk score, and status of patients. (C) Heatmap of the gene-expression Autophagy-related gene signature in GEO cohort.

A

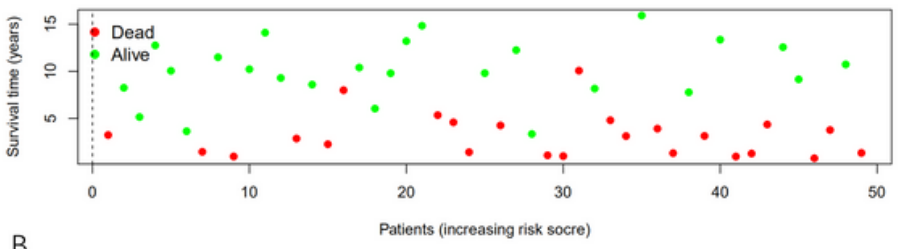

C

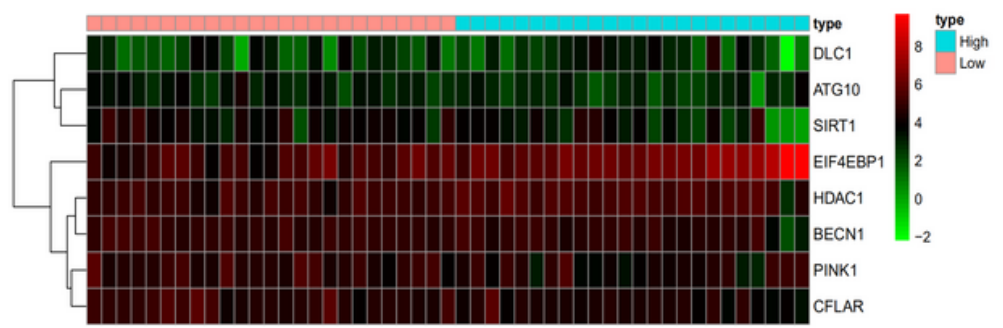

Figure 11

(A)The distributions of the survival time, and status of patients, (B)The distributions of the risk score, and status of patients. (C)Heatmap of the gene-expression Autophagy-related gene signature in ICGC cohort. 
A

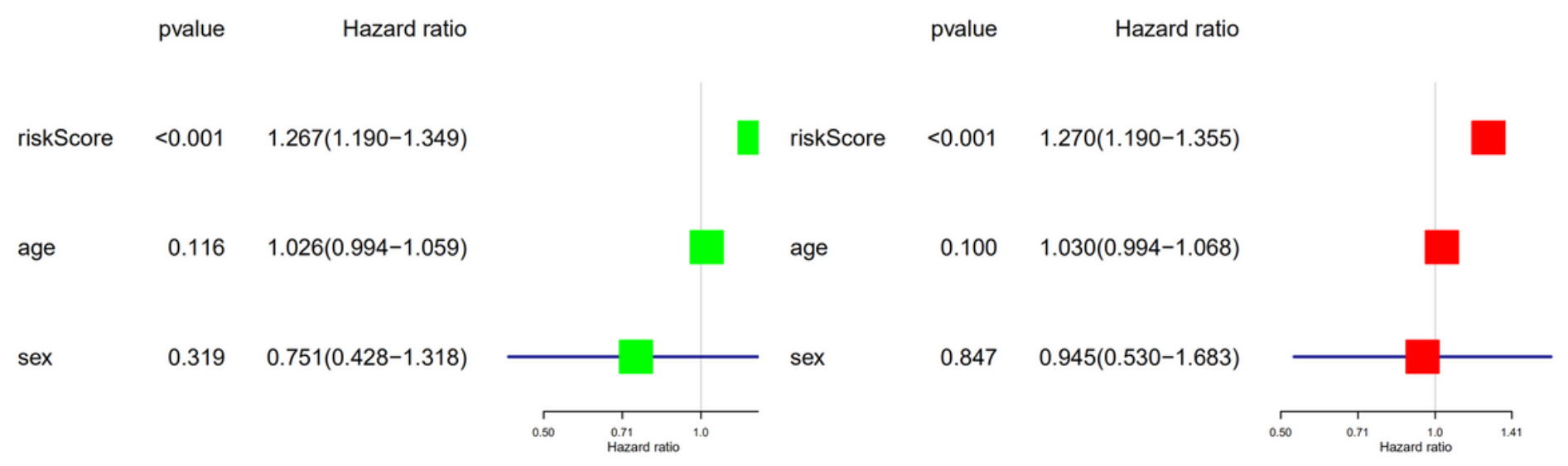

Figure 12

(A) univariate and (B) multivariate Cox regression analyses regarding OS.
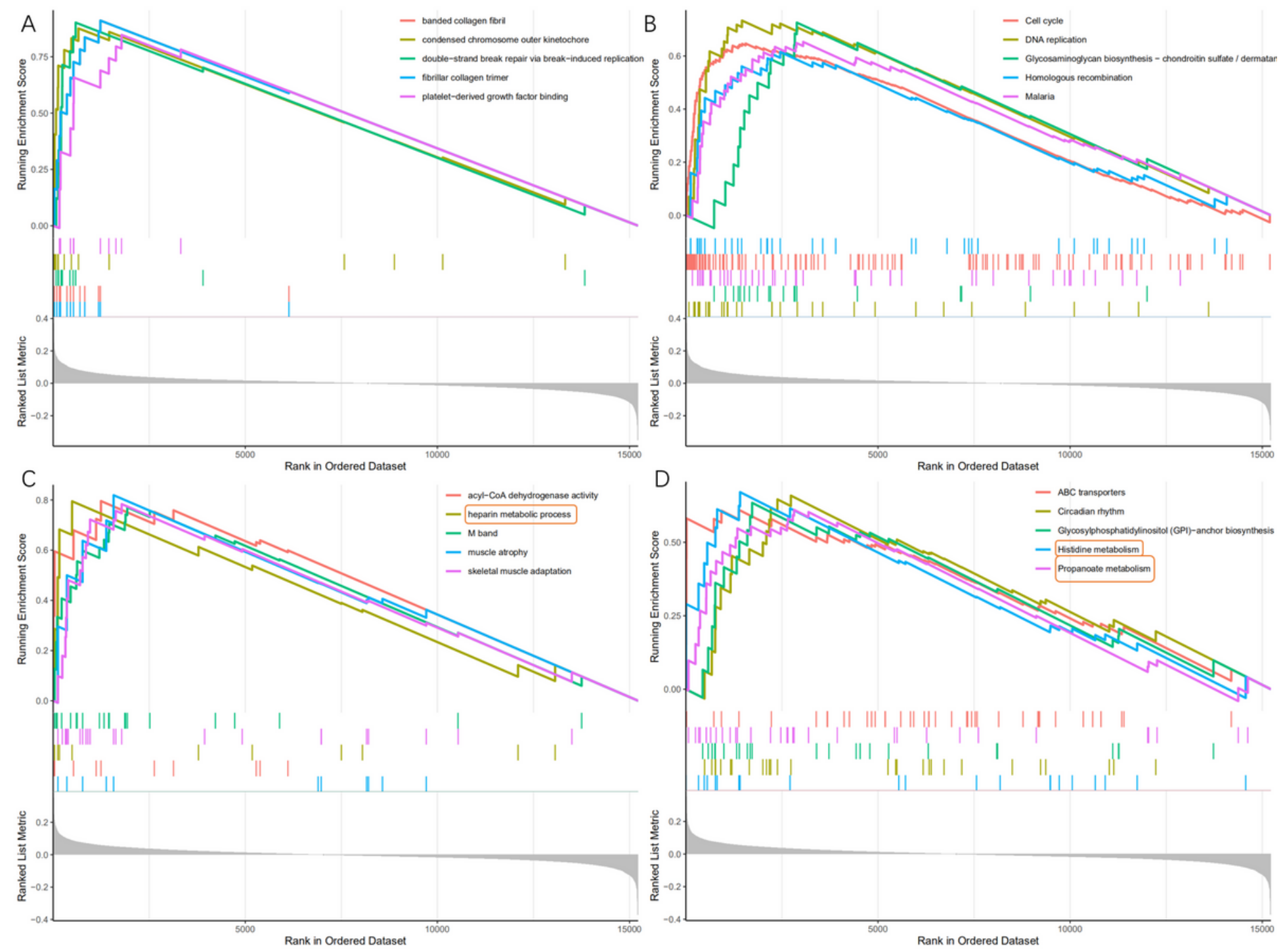

Figure 13 
$A$ and $B$ GO and KEGG GESA enrichment analysis in high-risk group. C and D GO and KEGG GSEA enrichment analysis in low-risk group.
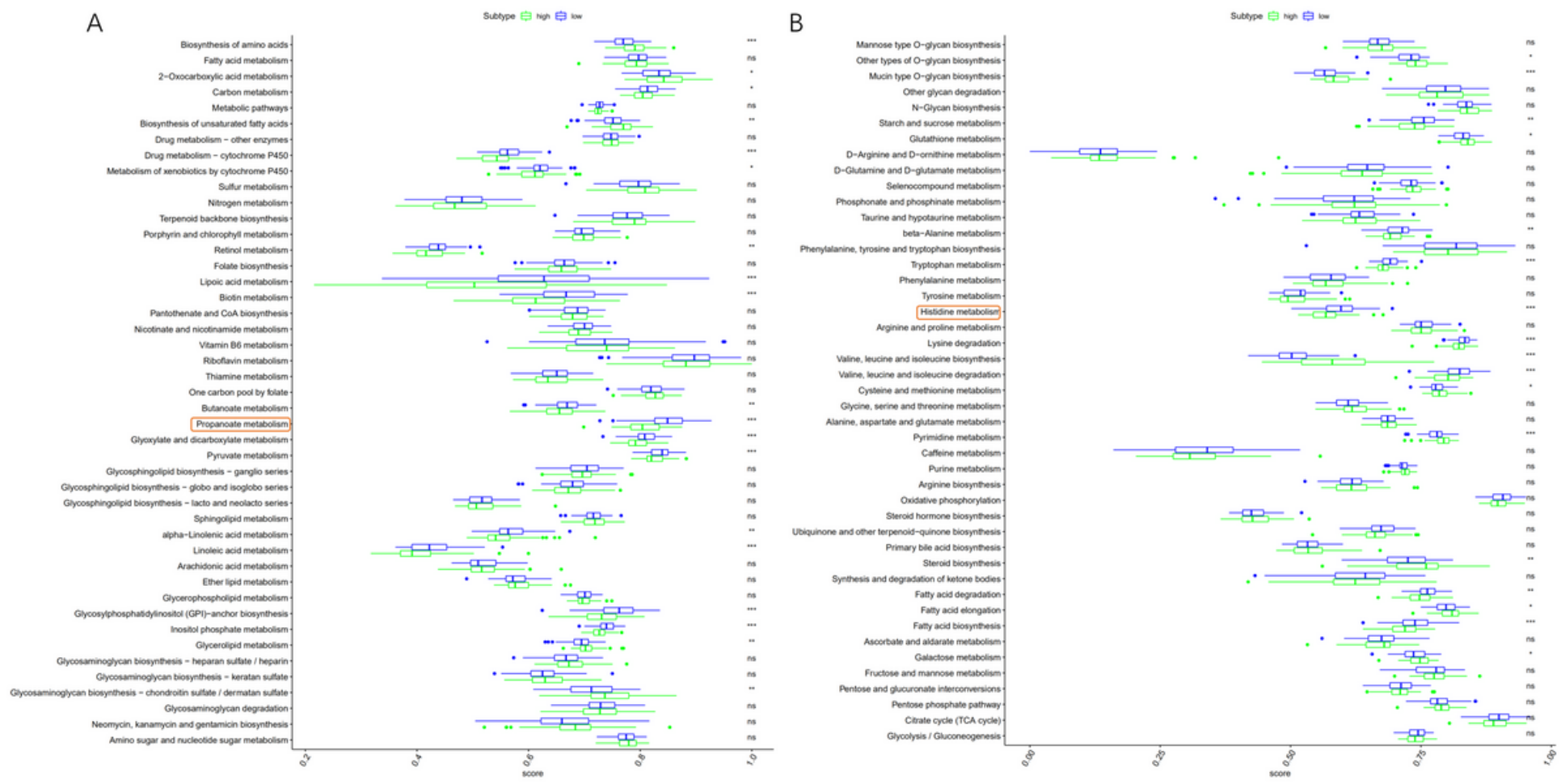

Figure 14

Comparison of the ssGSEA scores about metabolic pathways between different risk groups. ${ }^{*}, P<0.05$; $*$, $\mathrm{P}<0.01 ; * \star *, \mathrm{P}<0.001$. 